Revue de l’Université de Moncton

\title{
L'examen de l'article 37 de la Convention relative aux droits de l'enfant et des règles et principes des Nations Unies relatifs à la privation des libertés des enfants en conflit avec la loi
}

Revue de l'Université de Moncton

\section{Thierno Souleymane Barry}

Numéro hors-série, 2017

Enfants privés de liberté et justice pénale pour adolescents

URI : https://id.erudit.org/iderudit/1043659ar

DOI : https://doi.org/10.7202/1043659ar

Aller au sommaire du numéro

Éditeur(s)

Revue de l’Université de Moncton

ISSN

1712-2139 (numérique)

Découvrir la revue

Citer cet article

Barry, T. S. (2017). L'examen de l'article 37 de la Convention relative aux droits de l'enfant et des règles et principes des Nations Unies relatifs à la privation des libertés des enfants en conflit avec la loi. Revue de l'Université de Moncton, 83-104. https://doi.org/10.7202/1043659ar
Résumé de l'article

La lecture de l'article 37 de la Convention relative aux droits de l'enfant permet d'affirmer que l'enfant est protégé contre la torture et autres traitements cruels, inhumains et dégradants et est exempt de condamnation à la peine capitale et d'emprisonnement à vie sans possibilité de libération (art. 37.a), qu'il ne peut faire l'objet de privation illégale de liberté et que cette privation ne peut intervenir qu'en dernier ressort et de manière brève (art. 37.b), qu'en cas de privation de liberté, qu'il doit être traité avec dignité et séparé des adultes (art. 37.c) et qu'enfin, il doit bénéficier d'une assistance juridique et la garantie d'un procès équitable et rapide (art. 37. d). Ces principes rencontren les règles énoncées par les Nations unies : Règles de Beijing sur la justice des mineurs (1985), Principes de Riad sur la prévention de la délinquance juvénile (1990), Règles de La Havane sur la protection des mineurs privés de libertés (1990), Règles de Tokyo sur les mesures non privatives de libertés (1990).

En posant les principes d'exceptionnalité de la privation de liberté, de séparation des enfants et des adultes, de célérité de traitement, d'assistance juridique et de participation de l'enfant en conflit avec la loi, l'article 37 de la Convention relative aux droits de l'enfant et l'ensemble des corpus de règles et de principes des Nations Unies en la matière établissent un cadre de mise en oeuvre des droits de l'enfant privés de liberté compatible avec une approche des droits de l'enfant. Cependant, de plus en plus, pour juguler la délinquance juvénile, on constate un appel au durcissement des règles concernant le traitement des enfants en conflit avec la loi de la part des États et ce, en dépit d'un plaidoyer constant pour des mesures alternatives à la privation de la liberté des enfants en provenance des ONG et des comités onusiens.

Dans la présente étude, en faisant usage d'une approche basée sur les droits de l'enfant et en recourant aux dispositifs légaux, à la pratique des États et à la jurisprudence du Comité des droits de l'enfant, nous nous proposons d'analyser le contenu de l'article 37 de la Convention relative aux droits de l'enfant en lien avec les Règles et Principes des Nations Unies y afférant, d'évaluer la pratique des États et d'explorer les mesures alternatives relatives à la privation de la liberté des enfants en conflit avec la loi.
Ce document est protégé par la loi sur le droit d'auteur. L’utilisation des services d’Érudit (y compris la reproduction) est assujettie à sa politique d'utilisation que vous pouvez consulter en ligne.

https://apropos.erudit.org/fr/usagers/politique-dutilisation/ 
Revue de l'Université de Moncton, Numéro hors-série, 2017, p. 83-104.

L'EXAMEN DE L'ARTICLE 37 DE LA CONVENTION RELATIVE AUX DROITS DE L'ENFANT ET DES RÈGLES ET PRINCIPES DES NATIONS UNIES RELATIFS À LA PRIVATION DES LIBERTÉS DES ENFANTS EN CONFLIT AVEC LA LOI

\author{
Thierno Souleymane Barry \\ Université de Sonfonia, Conakry \\ et \\ Université de Sherbrooke
}

\title{
Résumé
}

La lecture de l'article 37 de la Convention relative aux droits de l'enfant permet d'affirmer que l'enfant est protégé contre la torture et autres traitements cruels, inhumains et dégradants et est exempt de condamnation à la peine capitale et d'emprisonnement à vie sans possibilité de libération (art. 37.a), qu'il ne peut faire l'objet de privation illégale de liberté et que cette privation ne peut intervenir qu'en dernier ressort et de manière brève (art. 37.b), qu'en cas de privation de liberté, qu'il doit être traité avec dignité et séparé des adultes (art. 37.c) et qu'enfin, il doit bénéficier d'une assistance juridique et la garantie d'un procès équitable et rapide (art. 37. d). Ces principes rencontrent les règles énoncées par les Nations unies : Règles de Beijing sur la justice des mineurs (1985), Principes de Riad sur la prévention de la délinquance juvénile (1990), Règles de La Havane sur la protection des mineurs privés de libertés (1990), Règles de Tokyo sur les mesures non privatives de libertés (1990).

En posant les principes d'exceptionnalité de la privation de liberté, de séparation des enfants et des adultes, de célérité de traitement, d'assistance juridique et de participation de l'enfant en conflit avec la loi, l'article 37 de la Convention relative aux droits de l'enfant et l'ensemble des corpus de règles et de principes des Nations Unies en la matière établissent un cadre de mise en œuvre des droits de l'enfant 
privés de liberté compatible avec une approche des droits de l'enfant. Cependant, de plus en plus, pour juguler la délinquance juvénile, on constate un appel au durcissement des règles concernant le traitement des enfants en conflit avec la loi de la part des États et ce, en dépit d'un plaidoyer constant pour des mesures alternatives à la privation de la liberté des enfants en provenance des ONG et des comités onusiens.

Dans la présente étude, en faisant usage d'une approche basée sur les droits de l'enfant et en recourant aux dispositifs légaux, à la pratique des États et à la jurisprudence du Comité des droits de l'enfant, nous nous proposons d'analyser le contenu de l'article 37 de la Convention relative aux droits de l'enfant en lien avec les Règles et Principes des Nations Unies y afférant, d'évaluer la pratique des États et d'explorer les mesures alternatives relatives à la privation de la liberté des enfants en conflit avec la loi.

Mots-clés : droits de l'enfant, enfants en conflit avec la loi, mesures non privatives de libertés, Règles et Principes des Nations Unies.

\begin{abstract}
The reading of Article 37 of the Convention on the rights of the child is to suggest that the child is protected against torture and other cruel, inhuman and degrading treatment and is free from condemnation to death and life imprisonment without possibility of release (Art. 37a), it may be illegal deprivation of liberty and that this deprivation can only intervene as a last resort and briefly (art. 37. b), in case deprivation of liberty, it must be treated with dignity and separated from adults (art. 37.c) and finally, it must have legal assistance and the guarantee of a fair and expeditious trial (art. 37. d). These principles meet the rules set by the United Nations: The Beijing Rules on Juvenile Justice (1985), Ryad Principles on the Prevention of Juvenile Delinquency (1990), The Havana Rules for the Protection of Juveniles Deprived of
\end{abstract}


their freedoms (1990), Tokyo Rules on non-custodial measures (1990).

By asking the exceptionality of the principles of the deprivation of liberty, separation of children and adults, speed of treatment, legal assistance and participation of children in conflict with the law, article 37 of the Convention on the rights of the child and all UN body of rules and principles in this area provide a framework for implementing the rights of the child deprived of liberty compatible with an approach to children's rights. However, increasingly, to curb juvenile delinquency, there is a call for tougher rules on the treatment of children in conflict with the law on the part of States and, despite a constant plea for alternative measures deprivation of liberty of children from NGOs and UN committees.

In this study, making use of an approach based on children's rights and resorting to legal measures, to state practice and jurisprudence of the Committee on the Rights of the Child, we propose to analyze the content of Article 37 of the Convention on the rights of the child in connection with the Rules and Principles of the United Nations relating to it, to assess the practice of States and explore alternative measures to deprivation of freedom of children in conflict with the law

Keywords: Children's Rights, children in conflict with the law, non-custodial measures, UN Rules and Principles.

\section{Introduction}

Face à la problématique des enfants en conflit avec la loi - et dans d'autres contextes - on assiste à une double préoccupation : la poursuite de la protection du bien-être de l'enfant et la réalisation de l'objectif de sécurisation de la société. De plus en plus, on remarque également l'existence d'un grand nombre d'enfants dans les liens de la justice et dans d'autres contextes. En effet, contrairement à ce qui est souvent véhiculé, le tableau de la situation des enfants privés de liberté déborde du cadre des enfants en conflit avec la loi et offre une variété de contextes : enfants en 
conflit avec la loi proprement dit, enfants en situation de trouble de santé mentale, enfants en situation d'immigration illégale, enfants en situation de placement... Toutes ces catégories d'enfant peuvent faire l'objet de privation de liberté et leur sort mérite d'être encadré au prisme de mesures alternatives à cette privation de liberté et avec pour base le respect de leurs droits.

Pour rester en accord avec les droits de l'enfant, les mesures privatives et non privatives de liberté de l'enfant doivent passer le test de l'approche basée sur les droits de l'enfant : une approche qui met l'enfant au centre de l'action ${ }^{1}$. Ainsi, les mesures privatives de liberté de l'enfant, qui doivent intervenir en dernier ressort, devraient obéir aux principes suivants : avoir un caractère exceptionnel, opérer la séparation de l'enfant et de l'adulte, prendre en compte l'intérêt de l'enfant et assurer la participation de l'enfant tout au long du processus. Quant aux mesures non alternatives et non privatives de liberté de l'enfant, outre certains aspects concernant les principes ci-dessus, elles doivent aussi, elles aussi, rencontrer les standards de respect de la dignité et de l'intégrité de l'enfant et de l'implication de l'enfant, entre autres.

Ces mesures sont contenues dans les dispositions pertinentes de la Convention relative aux droits de l'enfant (art. 37 et 40$)^{2}$ (ci-après «CIDE ») et les Règles et Principes des Nations Unies ${ }^{3}$; elles sont également rappelées par les Observations générales et finales du Comité des droits de l'enfant des Nations Unies ${ }^{4}$.

Ainsi, après avoir analysé l'article 37 de la CIDE (II) lu avec les Règles et les Principes de Nations Unies (III), nous présenterons le tableau des mesures alternatives à la privation de la liberté des enfants (IV) avec toujours en lien le respect des droits de l'enfant.

\section{Le contenu de l'article 37 de la CIDE lu avec les règles et principes des Nations Unies sur les mesures alternatives à la privation des libertés de l'enfant}

2.1. Le principe directeur de base : le droit de l'enfant de ne pas être privé de la liberté

Dans tous les contextes de privation de liberté, la règle de base demeure la soustraction de l'enfant des liens de l'incarcération en vue de préserver 
sa liberté, et ce, à tous les stades de la procédure ${ }^{5}$. Ce principe de ne pas être privé de liberté concerne toutes les formes de privation et suivant tous les contextes de privation de liberté : pénal, sanitaire, immigration illégale et autres. Le principe signifie que la liberté est la règle et la privation l'exception. Même dans un tel schéma, la privation de la liberté, pour être respectueuse des droits de l'enfant, doit être strictement encadrée et obéir à des règles claires; parmi lesquelles figurent le caractère révisable de la privation, sa temporalité, la recherche active de mécanismes alternatifs, etc.

Ce principe va au-delà des enfants en conflit avec la loi et concerne toutes les situations des enfants susceptibles de se voir privés de liberté. Pour illustration, concernant les enfants non accompagnés, le Comité des droits de l'enfant rappelle l'applicabilité du principe de mesures non privatives de la liberté de l'enfant avec vigueur dans son Observation générale No 06 en ces termes :

En application de l'article 37 de la Convention et du principe d'intérêt supérieur de l'enfant, les enfants non accompagnés ou séparés ne devraient pas, en règle générale, être placés en détention. La détention ne saurait être justifiée par le seul fait que l'enfant est séparé ou non accompagné ni par son seul statut au regard de la législation relative à l'immigration ou à la résidence ou l'absence d'un tel statut. Quand une détention se justifie à titre exceptionnel pour d'autres raisons, elle doit se dérouler conformément à l'article $37 \mathrm{~b}$ ) de la Convention qui dispose que la détention doit être en conformité avec la loi, n'être qu'une mesure de dernier ressort et être d'une durée aussi brève que possible. En conséquence, aucun effort ne devrait être négligé, notamment en vue de l'accélération de la procédure pertinente, pour permettre la libération immédiate d'un enfant non accompagné ou séparé retenu en détention et 
le placer dans un lieu d'hébergement approprié. [...]

En cas de détention, à titre de mesure exceptionnelle, les conditions de détention doivent être commandées par l'intérêt supérieur de l'enfant et respecter pleinement les alinéas $a$ et $c$ de l'article 37 de la Convention et les autres obligations internationales. Des dispositions spéciales doivent être prises pour mettre en place des quartiers adaptés aux enfants permettant de les séparer des adultes, à moins qu'il ne soit pas dans l'intérêt supérieur de l'enfant de procéder de la sorte. La démarche sous jacente d'un tel programme devrait être la " prise en charge » et non la « détention ${ }^{6}$.

Le principe peut trouver également application dans le domaine des enfants aux prises avec des troubles de santé mentale. Ces derniers ne doivent pas être arbitrairement privés de leur liberté. En cas de nécessité de leur mise en place dans un centre psychiatrique, l'ensemble de leurs droits doit être pris en compte et respecté. Il peut s'agir de prendre en compte leurs avis ou des personnes ayant la garde, de respecter leur intimité et de réévaluer leur situation de façon périodique. Ce principe est fermement rappelé à l'article 37 de la CIDE.

\subsection{La lecture de l'article 37 de la CIDE}

Les mesures concernant la liberté de l'enfant figurent à l'article 37 de la CIDE. Elle peut se lire en combinaison de l'article 40 de la même convention ${ }^{7}$. L'article 37 de la CIDE est libellé comme suit :

Les États parties veillent à ce que :

a) Nul enfant ne soit soumis à la torture ni à des peines ou traitements cruels, inhumains ou dégradants. $\mathrm{Ni}$ la peine capitale ni l'emprisonnement à vie sans possibilité de libération ne doivent être prononcés pour les infractions commises par des personnes âgées de moins de dix-huit ans; 
b) Nul enfant ne soit privé de liberté de façon illégale ou arbitraire. L'arrestation, la détention ou l'emprisonnement d'un enfant doit être en conformité avec la loi, n'être qu'une mesure de dernier ressort, et être d'une durée aussi brève que possible;

c) Tout enfant privé de liberté soit traité avec humanité et avec le respect dû à la dignité de la personne humaine, et d'une manière tenant compte des besoins des personnes de son âge. En particulier, tout enfant privé de liberté sera séparé des adultes, à moins que l'on estime préférable de ne pas le faire dans l'intérêt supérieur de l'enfant, et il a le droit de rester en contact avec sa famille par la correspondance et par les visites, sauf circonstances exceptionnelles;

d) Les enfants privés de liberté aient le droit d'avoir rapidement accès à l'assistance juridique ou à toute autre assistance appropriée, ainsi que le droit de contester la légalité de leur privation de liberté devant un tribunal ou une autre autorité compétente, indépendante et impartiale, et à ce qu'une décision rapide soit prise en la matière ${ }^{8}$.

La lecture de cette disposition nous fait ressortir les prescriptions qui suivent: l'interdiction de la torture, de la peine capitale et de l'emprisonnement à vie, l'interdiction de l'arrestation arbitraire et illégale, le caractère de dernier ressort et de brièveté de toute arrestation ou détention, le traitement humain et digne en cas d'emprisonnement, le principe de séparation de l'enfant et de l'adulte, le respect du droit à l'assistance et le principe de célérité dans les procédures concernant l'enfant.

L'insistance sur le respect de ces principes par les États revient dans plusieurs observations du Comité des droits de l'enfant des Nations Unies, dont celui portant sur le traitement des enfants non accompagnés et des enfants séparés en dehors de leur pays d'origine ${ }^{9}$. Cette disposition de l'article 37 de la CIDE est renforcée par l'apport des Règles et Principes des Nations Unies. 


\section{L'apport des règles et principes des Nations Unies}

\subsection{Les règles de Beijing ou l'ensemble de règles minima des Nations Unies concernant l'administration de la justice pour mineurs}

Les Règles de Beijing ${ }^{10}$, adoptées par l'Assemblée générale des Nations Unies à travers sa résolution 40/33 du 29 novembre 1985, concernent essentiellement les règles et principes de bases à observer pour une bonne administration de la justice pour les mineurs. Elles indiquent que la justice des mineurs est une partie intégrante " de la justice sociale des jeunes $»^{11}$. Les règles visent les jeunes déjà aux prises avec la justice et aux fins de ces règles, le mineur est défini comme : « $[\mathrm{u}] \mathrm{n}$ mineur est un enfant ou un jeune qui, au regard du système juridique considéré, peut avoir à répondre d'un délit selon des modalités différentes de celles qui sont appliquées dans le cas d'un adulte ${ }^{12}$. Un traitement équitable et en respect des droits fondamentaux de l'enfant est de mise. Quant à l'âge de responsabilité pénale, sans en fixer un seuil compte tenu de la diversité des systèmes juridiques, les règles indiquent, néanmoins, le souhait qu'il ne soit pas fixé trop bas ${ }^{13}$.

Les règles ont but principal le bien-être des mineurs comme le stipule son article 5. 1: «Le système de la justice pour mineurs recherche le bienêtre du mineur et fait en sorte que les réactions vis-à-vis des délinquants juvéniles soient toujours proportionnées aux circonstances propres aux délinquants et aux délits $»^{14}$. En raison de la qualité d'enfant en développement qui exige des solutions souples, il est prévu de faire recours à un large pouvoir discrétionnaire ${ }^{15}$ et responsable à toutes les étapes de la procédure visant les enfants en conflit avec la loi. En outre, le mineur doit se voir garantir l'ensemble des droits usuels en matière de justice équitable :

Les garanties fondamentales de la procédure telle que la présomption d'innocence, le droit à être informé des charges, le droit de garder le silence, le droit à l'assistance d'un conseil, le droit à la présence d'un parent ou tuteur, le droit d'interroger et de confronter les témoins et le droit à un double degré de juridiction sont assurées à tous les stades de la procédure $^{16}$. 
Enfin, tout au long de la procédure, la vie privée de l'enfant est strictement protégée non seulement contre la publicité, mais aussi contre la qualification pénale et la divulgation de son identité. Cette disposition demeure importante pour éviter de causer des dommages certains dans sa vie future. En matière d'instruction et de poursuites, il est indiqué que les procédures doivent avec célérité dès le premier contact de l'enfant avec la justice $^{17}$ et en recourant aux moyens extra judiciaires ${ }^{18}$. La détention préventive doit être en dernier ressort et de courte durée ${ }^{19}$. En matière de jugement et de règlement des affaires pénales, les principes de l'intérêt de l'enfant, de sa participation et de l'assistance d'un conseil demeurent en vigueur $^{20}$. La décision finale doit obéir à des principes directeurs stricts qui sauvegardent les droits et l'intérêt de l'enfant ${ }^{21}$. Tout doit être mis en œuvre pour éviter l'incarcération ${ }^{22}$ et le placement ${ }^{23}$ du mineur en institution. Les Règles proscrivent les délais inutiles en ces termes : "Toute affaire doit, dès le début, être traitée rapidement, sans retard évitable $»^{24}$. Les traces de jugement sont strictement règlementées afin de protéger le mineur ${ }^{25}$. À tous les stades, les personnes en contact avec les enfants doivent avoir une compétence et une formation avérée en la matière ${ }^{26}$. Dans le cadre de l'exécution de mesures privatives de liberté, il est souhaitable de faire recours au traitement en milieu ouvert ${ }^{27}$ et garder une possibilité de réhabilitation $^{28}$, avec l'appui de la communauté ${ }^{29}$. En cas de traitement en institution, l'enfant doit bénéficier des mesures supplémentaires de protection et parmi celles-ci figure en bonne place le fait d'être séparé des adultes $^{30}$. Durant tout son placement, l'Ensemble de règles minima pour le traitement des détenus adopté par l'Organisation des Nations Unies s'applique à l'enfant ${ }^{31}$. Parmi les panoplies des mesures allégeant la détention des mineurs telle la libération conditionnelle, figure en bonne place les régimes de semi-détention.

\subsection{Les Principes directeurs pour la prévention de la délinquance juvénile}

Les Principes directeurs des Nations Unies pour la prévention de la délinquance juvénile ${ }^{32}$, communément appelés Principes directeurs de Riad, furent adoptés par l'Assemblée générale des Nations Unies par la Résolution 45/112 du 14 décembre 1990. Comme leur nom l'indique, leur objectif est de prévenir la délinquance juvénile, il s'agit donc d'agir en amont en vue de prévenir tout facteur criminogène et éviter ainsi toute 
privation de la liberté de l'enfant à la source. Les Principes directeurs énoncent :

1. La prévention de la délinquance juvénile est un élément essentiel de la prévention du crime. En s'adonnant à des activités licites et utiles à la société et en se plaçant à l'égard de celle- ci et de la vie dans une perspective humaniste, les jeunes peuvent acquérir une mentalité non criminogène.

2. Pour que la prévention de la délinquance juvénile porte ses fruits, il faut que la société tout entière assure le développement harmonieux des adolescents en respectant leur personnalité et en favorisant l'épanouissement des jeunes dès la plus tendre enfance ${ }^{33}$.

Dans cette prévention, les jeunes sont au centre du processus comme l'indique le paragraphe 3 des Principes directeurs de Riad :

Aux fins de l'interprétation des présents
Principes directeurs, il conviendrait
d'adopter une orientation axée sur l'enfant.
Les jeunes devraient avoir un rôle actif de
partenaires dans la société et ne pas être
considérés comme de simples objets de
mesures de socialisation ou de contrôle ${ }^{34}$.

Sur la même lancée, il est indiqué que la prévention doit comprendre également l'exploration et l'usage des méthodes alternatives qui ne criminalisent pas les jeunes ${ }^{35}$.

Dans ce processus, on implique la famille, l'éducation et la communauté. Le placement en institution est de dernier ressort: "Le placement des jeunes en institutions devrait n'intervenir qu'en dernier ressort et ne durer que le temps absolument indispensable, l'intérêt de l'enfant étant la considération essentielle ${ }^{36}$. L'enfant est partie prenante de tout le processus du début à la fin. Enfin, pour leur mise en œuvre, les Principes directeurs de Riad encouragent l'installation d'un médiateur ${ }^{37}$ chargé de mettre en œuvre leurs prescriptions et d'assurer la protection de l'enfant. 
3.3. Les Règles de La Havane sur la protection des mineurs privés de libertés

Les Règles de La Havane sur la protection des mineurs privés de libertés $^{38}$ (1990) adoptées par l'Assemblée générale des Nations Unies par sa Résolution 45/113 du 14 décembre 1990, visent la protection de l'enfant qui a déjà privé de sa liberté. Elles posent dès le départ le caractère exceptionnel de l'incarcération des enfants en ces termes : « La justice pour mineurs devrait protéger les droits et la sécurité et promouvoir le bien-être physique et moral des mineurs. L'incarcération devrait être une mesure de dernier recours $»^{39}$.

Elles définissent la privation de la liberté de l'enfant comme : «[... toute forme de détention, d'emprisonnement ou le placement d'une personne dans un établissement public ou privé dont elle n'est pas autorisée à sortir à son gré, ordonnés par une autorité judiciaire, administrative ou autre ${ }^{40}$. En plus des droits usuels de base, il est prévu l'aménagement des établissements ouverts :

Des établissements ouverts pour mineurs doivent être créés. Les établissements ouverts sont des établissements dans lesquels les mesures matérielles de sécurité sont aussi réduites que possible. Dans de tels établissements, la population doit être assez restreinte pour permettre un traitement individualisé. Les établissements pour mineurs devraient être décentralisés et d'une taille propre à faciliter les contacts entre les mineurs et leurs familles. En particulier, on devrait créer de petits établissements de détention intégrés à l'environnement social, économique et culturel des mineurs et à leur communauté ${ }^{41}$.

Les Règles de La Havane rejoignent ainsi les principes et règles précédentes quant à la protection accrue des enfants en posant le principe de la préséance de l'insertion sur la répression. 
3.4. Les Règles de Tokyo sur les mesures non privatives de libertés

Les Règles minima des Nations Unies pour l'élaboration de mesures non privatives de liberté ${ }^{42}$, encore appelées les Règles de Tokyo, ont été adopté par l'Assemblée générale des Nations Unies à travers sa Résolution 45/110 du 14 décembre 1990. Les objectifs sont annoncés ainsi qu'il suit :

1.1 Les présentes Règles minima énoncent une série de principes fondamentaux en vue de favoriser le recours à des mesures non privatives de liberté ainsi que des garanties minima pour les personnes soumises à des mesures de substitution à l'emprisonnement.

1.2 Les présentes Règles visent à encourager la collectivité à participer davantage au processus de la justice pénale et plus particulièrement au traitement des délinquants ainsi qu'à développer chez ces derniers le sens de leur responsabilité envers la société.

1.3 L'application des présentes Règles tient compte de la situation politique, économique, sociale et culturelle de chaque pays et des buts et objectifs de son système de justice pénale.

1.4 Les États Membres s'efforcent d'appliquer les présentes Règles de façon à réaliser un juste équilibre entre les droits des délinquants, les droits des victimes et les préoccupations de la société concernant la sécurité publique et la prévention du crime.

1.5 Dans leurs systèmes juridiques respectifs, les États Membres s'efforcent d'élaborer des mesures non privatives de liberté pour offrir d'autres formules possibles afin de réduire le recours à 
l'incarcération, et pour rationaliser les politiques de justice pénale, eu égard au respect des droits de l'homme, aux exigences de la justice sociale et aux besoins de réinsertion des délinquants ${ }^{43}$.

Le traitement communautaire et non judiciaire est encouragé : « 2.5 On s'attachera, dans le respect des garanties juridiques et de la règle de droit, à traiter le cas des délinquants dans le cadre de la communauté en évitant autant que possible le recours à une procédure judiciaire ou aux tribunaux $\rangle^{44}$. Les mesures liées à la confidentialité, à la dignité et au respect des droits de l'enfant faisant objet de telles mesures sont protégées. S'inspirant de l'objectif poursuivi, les Règles établissent un quantum de peines respectueux des droits de l'enfant :

8.1 L'autorité judiciaire, ayant à sa disposition un arsenal de mesures non privatives de liberté, tient compte, dans sa décision, du besoin de réinsertion du délinquant, de la protection de la société et des intérêts de la victime, qui doit pouvoir être consultée toutes les fois que c'est opportun.

8.2 Les autorités compétentes peuvent prendre les mesures suivantes :

a) Sanctions orales, comme l'admonestation, la réprimande et l'avertissement;

b) Maintien en liberté avant décision du tribunal;

c) Peines privatives de droits;

d) Peines économiques et pécuniaires, comme l'amende et le jour-amende;

e) Confiscation ou expropriation;

f) Restitution à la victime ou indemnisation de celle-ci;

g) Condamnation avec sursis ou suspension de peine; 
h) Probation et surveillance judiciaire;

i) Peines de travail d'intérêt général;

j) Assignation dans un établissement ouvert;

k) Assignation à résidence;

1) Toute autre forme de traitement en milieu libre;

m) Une combinaison de ces mesures ${ }^{45}$.

Il apparait ainsi que dans la batterie des mesures proposées, les Règles de Tokyo s'inscrivent aussi dans celles précédentes de Riad, Havane et d'autres concernant la prise en compte des enfants susceptibles de privation de liberté. Ces Règles et Principes véhiculent des principes protégeant les enfants privés de liberté.

\section{Les principes véhiculés par les instruments internationaux et les recommandations du Comité des droits de l'enfant dans la mise en ouvre de l'article 37 de la CIDE}

\subsection{Les principes véhiculés}

Les principes véhiculés par ces ensembles de règles sont voisins sinon identiques à ceux de la CIDE. Les principes confirment l'affirmation du principe de l'exceptionnalité de la privation de la liberté de l'enfant. La poursuite du double respect du principe du bien-être et principe de la proportionnalité des délits et des peines constitue l'objectif principal.

En cas de privation de la liberté de l'enfant, l'aménagement de mesures protectrices doit être envisagé. Tant dans l'aménagement des endroits de privation de liberté de l'enfant que les activités qui s'y mènent doivent répondre à la protection de l'enfant. La garantie des droits fondamentaux des mineurs privés de liberté doit maintenue, la privation de la liberté n'a pas pour objectif la privation des droits de l'enfant. Parmi les droits de l'enfant qu'il faut garantir, figurent : l'information du mineur sur ses droits, le droit de se faire assister d'un avocat, le droit de maintenir de lien avec sa famille et toutes les autres garanties d'un procès équitable. 
En regard de la situation de l'enfant comme individu en développement et en respect de principe de développement, le respect du principe de célérité dans les procédures concernant les enfants doit être de mise. En effet, le temps chez l'enfant est très important et l'importance de cette notion de temps chez l'enfant a été soulignée par de nombreuses études ${ }^{46}$. $\mathrm{Ne}$ pas y souscrire et d'en accorder tout l'intérêt peut causer de dommages irréparables à l'enfant. C'est pour toutes ces raisons que les procédures entourant la privation de liberté de l'enfant doivent être aussi brèves que possible et menées avec une grande célérité. Dans le même ordre d'idées, les décisions entourant la privation de la liberté de l'enfant ne doivent pas être définitives et doivent être les plus flexibles possible. Il doit être envisagé la possibilité de révision à tout moment de la procédure.

Le principe de spécialité et de formation doit être également respecté de façon rigoureuse, au nom d'une mise en œuvre spécifique des droits de l'enfant. Ici également, l'enfant tout comme ses droits méritent une attention particulière. Cette protection spécifique due à l'enfant exige une formation particulière, et ce, tant dans le domaine juridique que dans les domaines psychosociaux et autres. Les juges des enfants, les travailleurs sociaux et le personnel pénitentiaire devraient être dotés d'une solide formation en psychologie de l'enfant, en droits de l'enfant, etc. de manière à bien agir dans la protection des droits de l'enfant. Ce n'est pas simplement un vœu formulé, mais une obligation des États qui est rappelée dans plusieurs instruments de protection des droits de l'enfant.

Enfin, dans toutes les affaires concernant la privation de la liberté de l'enfant, préférence doit être accordée à la réhabilitation de l'enfant en conflit avec la loi au lieu de son incarcération. Quand bien même cette option demeure la dernière et seule solution, la réhabilitation de l'enfant est rigoureusement recommandée. Parmi les mesures favorisant son insertion, on peut noter les centres semi-fermés, les centres en milieu ouvert, les centres d'apprentissage, etc. Au regard de ce qui précède, il serait intéressant d'examiner la pratique effective des États dans la mise en œuvre de l'article 37 de la CIDE et des Règles et Principes des Nations Unies. 


\subsection{Les Recommandations du Comité des droits de l'enfant des Nations Unies}

Le Comité des droits de l'enfant des Nations Unies a indiqué, à travers son Observation générale No $10(2007)^{47}$, les démarches à suivre pour des bonnes pratiques en matière de mesures alternatives à la privation de la liberté des enfants. Il pose les principes de prévention de la délinquance juvénile et la déjudiciarisation des affaires impliquant les enfants :

Les autorités de l'État peuvent employer deux types d'intervention pour traiter les enfants suspectés, accusés ou convaincus d'infraction à la loi pénale: les mesures ne recourant pas à la procédure judiciaire et les mesures s'inscrivant dans le cadre de la procédure judiciaire.

Le Comité rappelle aux États parties qu'il faut s'attacher avec le plus grand soin à faire respecter et protéger pleinement les droits fondamentaux de l'enfant et les garanties juridiques en sa faveur ${ }^{48}$.

Sur la même lancée, les États sont invités à intégrer des mécanismes ${ }^{49}$ permettant à la fois le non-recours à la procédure judiciaire et le respect des droits fondamentaux des droits de l'enfant :

Les États parties devraient intégrer dans leur système de justice pour mineurs des mesures pour traiter les enfants en conflit avec la loi sans recourir à la procédure judiciaire et veiller à faire pleinement respecter et protéger les droits fondamentaux de ces enfants et les garanties juridiques en leur faveur (art. 40 3) b) $)^{50}$.

Ces mesures non judiciaires devraient: «[...] englober les soins, l'orientation et la supervision, les conseils, la probation, le placement familial et les programmes d'éducation générale et professionnelle, ainsi que diverses solutions autres qu'institutionnelles (art. 404$)) »^{51}$. 
En prenant seulement pour appui les diverses constatations et les observations finales du Comité des droits de l'enfant des Nations Unies, on peut relever quelques constats soulevés dans la pratique des États : un âge de responsabilité très bas ou confusion autour de l'âge de responsabilité, la persistance de non séparation des enfants et des adultes, notamment en ce qui concerne les jeunes filles, les mauvais traitements dans les prisons, l'absence des mesures alternatives à la privation de la liberté des enfants... À ces manquements, il serait souhaitable que les États procèdent à une série de mesures dont le relèvement de l'âge de responsabilité pénale pour l'enfant, l'absence de la torture et des mauvais traitements aux enfants, la séparation des enfants et des adultes...

\section{Présentation des mesures alternatives à la privation de la liberté des enfants en lien avec le respect des droits de l'enfant}

\subsection{La réhabilitation au sein de la communauté}

Dans la batterie de mesures concernant les alternatives à la privation de la liberté de l'enfant, le maintien au sein de la communauté demeure une action salvatrice. Le Comité des droits de l'enfant des Nations Unies réitère d'impliquer la communauté dans le processus de réhabilitation de l'enfant en conflit avec la 1 loi $^{52}$. Il peut s'agir des travaux au bénéfice de la communauté, d'encadrement communautaire, des centres de formation professionnelle gérés par la communauté... Pour les Principes directeurs de Riad :

Il faudrait que la communauté mette en place,
ou renforce, s'il en existe déjà, des moyens
très variés d'assistance communautaire aux
jeunes tels que des centres de développement
communautaire, équipements récréatifs et
services conçus en fonction des problèmes
spéciaux des enfants en situation de «risque
social ». Il faudrait veiller, ce faisant, à
respecter les droits de l'individu ${ }^{53}$.

On peut aussi initier des actions intergénérationnelles visant à associer les personnes âgées et les enfants dans le but de transmission des valeurs positives de la communauté ou de léguer des savoirs traditionnels positifs 
(savoirs agricoles, artisanaux...). Ces mesures permettent une intégration de l'enfant dans la communauté et de lui permettre de jouer un rôle d'acteur au sein de cette même communauté.

\subsection{La participation de l'enfant dans la réalisation des mesures de réhabilitation}

L'une des innovations majeures de la Convention relative aux droits de l'enfant est constituée par la disposition de l'article 12 qui fait de la participation de l'enfant un principe d'interprétation des droits qu'elle reconnaisse aux enfants. Elle est ainsi libellée :

1. Les États parties garantissent à l'enfant qui est capable de discernement le droit d'exprimer librement son opinion sur toute question l'intéressant, les opinions de l'enfant étant dûment prises en considération eu égard à son âge et à son degré de maturité.

2. À cette fin, on donnera notamment à l'enfant la possibilité d'être entendu dans toute procédure judiciaire ou administrative l'intéressant, soit directement, soit par l'intermédiaire d'un représentant ou d'une organisation approprié, de façon compatible avec les règles de procédure de la législation nationale ${ }^{54}$.

Selon le Comité des droits de l'enfant des Nations Unies, la participation de l'enfant est un des principes d'interprétation des tous les autres droits de l'enfant. Il est aussi conforme à l'approche basée sur les droits de l'enfant qui met l'enfant au centre du processus de protection de ses droits ${ }^{55}$. Il est à noter que cette participation de l'enfant doit s'étendre à toutes les questions concernant l'enfant ${ }^{56}$.

Ainsi, les décisions concernant les mesures de réhabilitation de l'enfant doivent être prises avec l'implication de l'enfant dans toutes les phases du processus : la décision d'y recourir, le choix des mesures, leur nature, leur durée, leur modalité d'exécution, etc. Les Principes directeurs des Nations Unies pour la prévention de la délinquance juvénile énoncent, à cet effet que « [d]ans l'ensemble, la participation aux plans et programmes devrait être volontaire, et il faudrait que les jeunes eux-mêmes prennent part à la conception, à l'élaboration et à l'exécution de ces plans et programmes $»^{57}$. 
5.3. Tableau des mesures alternatives non privatives des libertés de l'enfant et respectueuses de ses droits à explorer

Il peut exister plusieurs mesures alternatives à la privation de la liberté de l'enfant. Ces mesures peuvent s'appliquer de manière autonome ou en combinaison avec d'autres mécanismes de même nature. L'ensemble de ces mesures doit s'effectuer dans le respect de la vie privée de l'enfant et de ses autres droits comme l'indiquent les règles Beijing :

8.1 Le droit du mineur à la protection de sa vie privée doit être respecté à tous les stades afin d'éviter qu'il ne lui soit causé du tort par une publicité inutile et par la qualification pénale.

8.2 En principe, aucune information pouvant conduire à l'identification d'un délinquant juvénile ne doit être publiée ${ }^{58}$.

Les régimes de centres semi-fermés sont privilégiés par les Règles de Beijing :

On s'efforcera de créer des régimes de semidétention notamment dans des établissements tels que les centres d'accueil intermédiaires, les foyers socio-éducatifs, les externats de formation professionnelle et autres établissements appropriés propres à favoriser la réinsertion sociale des mineurs ${ }^{59}$.

Les mesures impliquent une professionnalisation des intervenants à l'intérieur des centres fermés accueillant les enfants en conflit avec la loi. Les enfants placés dans des centres fermés pour des durées plus ou moins longues peuvent apprendre un métier. La combinaison de la détention et la pratique d'un métier permettront de résoudre parfois la criminalité qui est parfois causée par l'inactivité et le manque de débouché pour le jeune en conflit avec la loi. Lors qu'en sortant de la détention, le jeune en conflit avec la loi est nanti d'une formation et qu'un suivi régulier est effectué auprès de lui, il peut s'insérer très aisément dans la communauté. Les 
mesures en semi-liberté sont aussi de nature à favoriser l'insertion de l'enfant susceptible d'être privé de liberté.

\section{Conclusion}

En somme, en vertu de la Convention relative aux droits de l'enfant et des Règles et Principes des Nations Unies en la matière, la privation de la liberté de l'enfant demeure une exception strictement encadrée. En raison de sa vulnérabilité et dans le but de protéger ses droits et son intérêt, l'accent doit être mis sur la prévention et l'insertion de l'enfant en conflit avec la loi au sein de la communauté.

1 Tun Aye Aye et al., « The domestic fulfilment of children's rights: save the children's experience in the use of rights-based approaches » dans A. Alen (dir.), The United Nations children's rights convention: theory meets practice (Proceedings of the International and interdisciplinary conference on children's rights, 18-19, May 2006, Ghent, Belgium), Oxford, Intersentia, 2007, 34.

2 Convention relative aux droits de l'enfant, New York, 20 novembre 1989, 1577 RTNU 3.

3 Office des Nations Unies contre la drogue et le crime, Recueil des règles et normes de l'Organisation des Nations Unies en matière de prévention du crime et de justice pénale, New York, 2007.

4 Pour les Observations générales et finales du Comité des droits de l'enfant des Nations Unies, voir en ligne: <www.unicef.ca/fr/discover-fr/article/observations-generales-interpretation-de-laconvention>.

5 Convention relative aux droits de l'enfant, supra note 2, art 37(b).

6 Comité des droits de l'enfant, «Observation générale No. 6: Traitement des enfants non accompagnés et des enfants séparés en dehors de leur pays d'origine » U.N. Doc. CRC/GC/ 2005/6 (2005), para 61 et 63 .

7 Convention relative aux droits de l'enfant, supra note 2, art 40(1) : « Les États parties reconnaissent à tout enfant suspecté, accusé ou convaincu d'infraction à la loi pénale le droit à un traitement qui soit de nature à favoriser son sens de la dignité et de la valeur personnelle, qui renforce son respect pour les droits de l'homme et les libertés fondamentales d'autrui, et qui tienne compte de son âge ainsi que de la nécessité de faciliter sa réintégration dans la société et de lui faire assumer un rôle constructif au sein de celle-ci ».

8 Convention relative aux droits de l'enfant, supra note 2, art 37.

9 Comité des droits de l'enfant, Observation générale No 6, supra note 6.

10 Assemblée générale des Nations Unies, Ensemble de règles minima des Nations Unies concernant l'administration de la justice pour mineurs (Règles de Beijing), AGNU Rés 40/33, 29 novembre 1985.

$11 \quad$ Ibid, art 1.4.

12 Ibid, art 2.2(a). 
13 Ibid, art 4.1 : «Dans les systèmes juridiques qui reconnaissent la notion de seuil de responsabilité pénale, celui-ci ne doit pas être fixé trop bas eu égard aux problèmes de maturité affective, psychologique et intellectuelle ».

Ibid, art 5.1.

$15 \quad$ Ibid, art 6.

16 Ibid, art 7.1.

17 Ibid, art 10.

18 Ibid, art 11.

19 Ibid, art 12.

20 Ibid, art 14 et suiv.

$21 \quad$ Ibid, art. 17.

22 Ibid, art 18.

23 Ibid, art 19.

24 Ibid, art 20.1.

25 Ibid, art 21.

26 Ibid, art 22.

27 Ibid, art 23.

28 Ibid, art 24.

29 Ibid, art. 25.

$30 \quad$ Ibid, art. 26.3.

31 Ibid, art 27.

32 Assemblée générale des Nations Unies, Les Principes directeurs des Nations Unies pour la prévention de la délinquance juvénile, AGNU Rés 45/112, 14 décembre 1990.

33 Ibid, para 1 et 2 .

$34 \quad$ Ibid, para 3.

$35 \quad$ Ibid, para 5.

$36 \quad$ Ibid, para 46.

37 Ibid, para 57.

38 Assemblée générale des Nations Unies, Règles de La Havane sur la protection des mineurs privés de libertés, AGNU Rés 45/113, 14 décembre 1990.

$39 \quad$ Ibid, para 1.

$40 \quad$ Ibid, para 11 b).

41 Ibid, para 30.

42 Assemblée générale des Nations Unies, Règles minima des Nations Unies pour l'élaboration de mesures non privatives de liberté, AGNU Rés 45/110, 14 décembre 1990.

$43 \quad$ Ibid, para 1.

44 Ibid, para 2.5. 
104 Revue de l'Université de Moncton, Numéro hors-série, 2017

$45 \quad$ Ibid, para 8.

46 Sur la notion du temps chez l'enfant, voir notamment Valérie Tartas, «Le développement des notions temporelles par l'enfant» (2010) I:4 Développements 17.

47 Comité des droits de l'enfant, «Observation générale no 10 (2007) : Les droits de l'enfant dans le système de justice pour mineurs, $\mathrm{CRC} / \mathrm{C} / \mathrm{GC} / 10,25$ avril 2007.

48 Ibid, para 22.

49 Cependant, ces mécanismes tardent à être suivis par certains États ; voir Comité des droits de l'enfant, «Examen des rapports présentés par les États parties en applications de l'article 44 de la Convention: Observations finales - République dominicaine », $\mathrm{CRC} / \mathrm{C} / \mathrm{DOM} / \mathrm{CO} / 2,11$ février 2008. À cet effet, le Comité des droits de l'enfant a fait à la République dominicaine les recommandations suivantes: «a) De prendre toutes les mesures nécessaires, en adoptant notamment une politique permanente de peines de substitution pour les jeunes délinquants, afin de garantir que les enfants ne soient placés en détention qu'en dernier ressort et pour une durée aussi courte que possible; b) De veiller à ce que la détention soit exécutée conformément aux articles 37 et 40 de la Convention et à ce que les enfants ne soient pas maltraités, que leur développement ne soit pas menacé par les conditions régnant dans les centres de détention et que leurs droits ne soient pas violés »; Ibid à la p 20.

50 Comité des droits de l'enfant, Observation générale no 10, supra note 47, para 26.

51 Ibid, para 23.

52 Ibid à la $\mathrm{p} 17$.

53 Principes directeurs des Nations Unies pour la prévention de la délinquance juvénile, supra note 32, para 33.

54 Convention relative aux droits de l'enfant, supra note 2.

55 Mark Ensalaco et Linda C. Majka, «Introduction: a human rights approach to the needs of children » dans Mark Ensalaco et Linda C. Majka (dir.), Children's human rights, Lanham, Rowman \& Littlefield, 2005, 2.

56 Patrick Parkinson, « The child participation principle in child protection law in New South Wales » (2001) 9 Int'1 J. Child. Rts 259 à la p 259.

57 Principes directeurs des Nations Unies pour la prévention de la délinquance juvénile, supra note 32 , para 50.

58 Ensemble de règles minima des Nations Unies concernant l'administration de la justice pour mineurs, supra note 10 , art 8.

59 Ibid, art 29.1. 